\title{
Distrubution of Extended Spectrum $\beta$-Lactamase and AMPC- $\beta$ Lactamase Among Bacteria Isolated From Urine Samples
}

\author{
${ }^{1}$ Bishal Shrestha, ${ }^{2}$ Jyoti Acharya, ${ }^{3}$ Jyoti Chhetri ${ }^{3}$ Krishna Gurung,${ }^{3}$ Mamita Khaling Rai \\ ${ }^{1}$ Nepal Pharamaceuticals Lab. Pvt Ltd, Birgunj, \\ ${ }^{2}$ National Public Health Laboratory, Kathmandu, \\ ${ }^{3}$ Pokhara Bigyan Tatha Prabidhi Campus, Pokhara, Nepal.
}

\begin{abstract}
This study was designed to determine the distribution of bacterial strains isolated from patients with urinary tract infections (UTI) at Sukrararj Tropical and Infectious Disease Hospital (STIDH) with special reference to ESBL, ABL and the co-existence of the phenotype (ESBL and ABL in the population of different uropathogens. Urine samples from 500 patients suspected of UTI were analyzed by conventional semi-quantitative culture technique. The antimicrobial susceptibility to various drugs was studied by the Modified Kirby-Bauer disk diffusion method, by following the CISI guidelines. Confirmation of the extended spectrum $\beta$-lactamase (ESBL) and AmpC $\beta$-lactamase production was done by the phenotypic confirmatory disc diffusion test (PCDDT) and the AmpC disk test (ADT). Overall, the prevalence of ESBL and ABL and the co-existence of the phenotype ( $E S B L$ and $A B L$ ) in urinary isolates were found to be $39.22 \%, 15.69 \%$ and $5.88 \%$. The implementation of appropriate infection control measures and the formulation of an antibiotic policy must be done, to prevent the spread of these strains.
\end{abstract}

Key words: $M D R, E S B L s . A m p C \beta$-lactamase

Corresponding address: Bishal Shrestha, M.Sc Medical Microbiology, Nepal Pharmaceutical Lab. Pvt.Ltd, Birgunj, E-mail: bishalshrestha001@gmail.com.

\section{INTRODUCTION}

The production of Beta-lactamases is an important mechanism of resistance to Beta-lactam antibiotics among gram negative bacteria. ${ }^{1}$ Extended-Spectrum-Beta-Lactamases (ESBLs) are plasmid-mediated beta-lactamases of predominantly Bush class A, so far described only in gram negative bacilli. ESBLs are mutants of TEM-1, TEM-2 and SHV-1, that are capable of efficiently hydrolyzing penicillin, narrow spectrum cephalosporins (e.g. cefotaxime, ceftazidine) and monobactams (e.g aztreonam), but they do not hydrolyze cephamycin or carbapenems (imipenem, meropenem). ${ }^{2}$ BetaLactamase inhibitors such as clavulanic acid, sulbactam and tazobactam generally inhibit ESBL producing strains. ESBL producing isolates are most commonly found in Klebsiella spp, (predominantly Klebsiella pneumoniae) and E. coli. ${ }^{3}$

AmpC $\beta$-lactamases preferentially hydrolyze narrow, broad, and expanded-spectrum cephalosporins and cephamycins and resist inhibition by clavulanate, sulbactam, and tazobactam. Many gram-negative bacilli produce a chromosomally mediated AmpC which, when hyperproduced, may cause resistance to penicillins, aztreonam, cephamycins, and narrow-, broad-, and expanded-spectrum cephalosporins. Transmissible AmpC $\beta$-lactamases are referred as plasmidmediated AmpC $\beta$-lactamases. These enzymes have been detected in some isolates of Klebsiella spp., Salmonella spp., C. freundii, E. aerogenes, P. mirabilis, and E. coli and are typically associated with multidrug resistance. The most commonly encountered plasmid-mediated AmpC $\beta$-lactamases belong to the CMY, FOX, and DHA families. Accurate prevalence data are scarce due to lack of testing, but they appear to be less common than ESBLs. ${ }^{4}$

Antibiotic treatment is typically selected empirically, based on the patient clinical presentation, medical history and local patterns of antibiotic susceptibility. ${ }^{5}$ In modern medical practice, newer antimicrobial drugs have been used extensively resulting in emergence and rapid dissemination of resistant bacterial strains. ${ }^{6}$ Hence this study was conducted to determine the distribution of bacterial strains isolated from patients with urinary tract infections (UTI) at Sukrarj Tropical and Infectious Disease Hospital (STIDH) with special reference to ESBL, Amp C and co-existence of ESBL and ABL production in Gram negative population of different uropathogens.

\section{MATERIALS AND METHODS}

A total of 500 urine samples from patients suspected of UTI were collected and processed according to the standard laboratory methods.

ESBL Screening and Confirmation

The isolates were tested for their susceptibility to the third generation cephalosporins (3GCs) ceftazidime $(30 \mu \mathrm{g})$, cefotaxime $(30 \mu \mathrm{g})$ and ceftriaxone $(30 \mu \mathrm{g})$ by using the standard disc diffusion method, as was recommended by the CLSI. ${ }^{7}$ If a zone diameter of $<22 \mathrm{~mm}$ for ceftazidime, $<$ $27 \mathrm{~mm}$ for cefotaxime and $<25 \mathrm{~mm}$ for ceftriaxone were recorded, the strain was considered to be "suspicious for 
ESBL production”. Only those isolates which were resistant to one of the $3 \mathrm{GCs}$ were selected for the study and they were processed for the ESBL production.

Phenotypic confirmatory dise diffusion test (PCDDt): All the strains which were screened out for the ESBL production were subjected to confirmation by using the PCDDT, as was recommended by the CLSI. In this test, ceftaxime (30 $\mu \mathrm{g})$ discs alone and in combination with clavulanic acid (cefotaxime + clavulanic Acid, 30/10 $\mu \mathrm{g}$ ) discs, were applied onto a plate of Mueller Hinton Agar (MHA) which was inoculated with the test strain. An increase of $\geq 5 \mathrm{~mm}$ in the zone of inhibition of the combination discs in comparison to that of the cefotaxime disc alone was considered to be a marker for ESBL production. ${ }^{7,8}$

AmpC screening and confirmation:

\section{AmpC Screening Test}

The susceptibility of the isolates to cefoxitin disc $(30 \mu \mathrm{g})$ was determined by the standard Disc Diffusion method. Isolates that yielded a cefoxitin zone diameter less than $18 \mathrm{~mm}$ were tested for AmpC enzyme production by AmpC disk test. ${ }^{3,9,10,11,12}$

\section{Confirmatory Test (AmpC Disk Test)}

The cefoxitin (CX) $(30 \mu \mathrm{g})$ and cefoxitin/phenylboronic acid $(\mathrm{CX} / \mathrm{BA})(30 / 400 \mu \mathrm{g})$ was placed on the Mueller Hinton agar (Hi-media, Mumbai) containing 0.5 McFarland turbid test culture. After the incubation period of 16-18hours at $37^{\circ} \mathrm{C}$, the isolates with inhibition zone difference of $5 \mathrm{~mm}$ between CX/BA and CX alone was considered as AmpC producers. The boronic acid was prepared by dissolving the $120 \mathrm{mg}$ of phenylboronic acid in $3 \mathrm{ml}$ of dimethyl sulfoxide (DMSO) and $3 \mathrm{ml}$ of sterile distilled water was added to this solution. Finally $20 \mu \mathrm{l}$ of stock solution was dispensed on to cefoxitin disc $(30 \mathrm{mcg})$. Discs were allowed to dry for 45-60 minutes and used immediately or stored in airtight vials with desiccant at $4^{0} \mathrm{C} .1,9,11$

\section{Data analysis}

All the results were entered in the worksheet of SPSS software (version 16.0).

\section{RESULTS}

Table 1: Distribution of ESBL production in gram negative isolates.

\begin{tabular}{|c|c|c|}
\hline \multirow{2}{*}{ Gram negative isolates } & \multicolumn{2}{|c|}{ ESBL Type } \\
\hline & ESBL producer & Non- ESBL producer \\
\hline Escherichia coli & $17 / 32(53.13 \%)$ & $15 / 32(46.87 \%)$ \\
\hline Klebsiella pneumonia & $1 / 4(25 \%)$ & $3(75 \%)$ \\
\hline Klebsiella oxytoca & $0 / 2(0 \%)$ & $2 / 2(100 \%)$ \\
\hline Acinetobacter spp & $0 / 5(0 \%)$ & $5 / 5(100 \%)$ \\
\hline Pseudomonas aeruginosa & $1 / 3(33.33 \%)$ & $2 / 3(66.67 \%)$ \\
\hline Citrobacter freundii & $0 / 1(0 \%)$ & $1 / 1(100 \%)$ \\
\hline Citrobacter diversus & $0 / 3(0 \%)$ & $3 / 3(100 \%)$ \\
\hline Proteus vulgaris & $1 / 1(100 \%)$ & $0(0 \%)$ \\
\hline Total & $20 / 51(39.22 \%)$ & $31 / 51(60.78 \%)$ \\
\hline
\end{tabular}

Out of 51 Gram negative isolates, 20 isolates (39.22\%) were ESBL producers. The maximum ESBL activity was seen in Proteus vulgaris (100 \%), followed by Escherichia coli (53.13\%), Pseudomonas aeruginosa (33.33\%) and Klebsiella pneumoniae (25\%). No ESBL activity was seen in Klebsiella oxytoca, Acinetobacter spp, Citrobacter freundii and Citrobacter diversus.

Table 2: Distribution of AmpC beta-lactamase producer in gram negative isolates.

\begin{tabular}{|l|l|l|}
\hline \multirow{2}{*}{ Gram negative isolates } & \multicolumn{2}{|c|}{ AmpC Type } \\
\cline { 2 - 3 } & AmpC producer & Non-AmpC producer \\
\hline Escherichia coli & $8 / 32(25 \%)$ & $24 / 32(75 \%)$ \\
\hline Klebsiella pneumonia & $0 / 4(0 \%)$ & $4 / 4(100 \%)$ \\
\hline Klebsiella oxytoca & $0 / 2(0 \%)$ & $2 / 2(100 \%)$ \\
\hline Acinetobacter spp & $0 / 5(0 \%)$ & $5 / 5(100 \%)$ \\
\hline Pseudomonas aeruginosa & $0 / 3(0 \%)$ & $3 / 3(100 \%)$ \\
\hline Citrobacter freundii & $0 / 1(0 \%)$ & $1 / 1(100 \%)$ \\
\hline Citrobacter diversus & $0 / 3(0 \%)$ & $3 / 3(100 \%)$ \\
\hline Proteus vulgaris & $0 / 1(0 \%)$ & $1 / 1(100 \%)$ \\
\hline \multicolumn{1}{|c|}{ Total } & $8 / 51(\mathbf{1 5 . 6 9} \%)$ & $\mathbf{4 3 / 5 1 ( 8 4 . 3 1 \% )}$ \\
\hline
\end{tabular}

Out of 51 Gram negative isolates, 8 isolates $(15.69 \%)$ were Ampc beta lactamase producers. The maximum Ampc beta lactamase production was found in Escherichia coli (25\%). No Ampc beta lactamase production activity was found in Klebsiella pneumoniae, Klebsiella oxytoca, Acinetobacter spp, Pseudomonas aeruginosa, Citrobacter freundii, Citrobacter diversus and Proteus vulgaris.

Table 3: Distribution of ESBL, ABL and co-existence of ESBL and $A B L$ production in Gram negative isolates

\begin{tabular}{|l|l|l|l|}
\hline \multicolumn{1}{|c|}{ Bacterial isolates } & \multicolumn{1}{|c|}{ ESBL } & \multicolumn{1}{|c|}{ AmpC } & \multicolumn{1}{c|}{$\begin{array}{c}\text { ESBL } \\
+ \text { AmpC }\end{array}$} \\
\hline Escherechia coli & $17 / 32(53.13 \%)$ & $8 / 32(25 \%)$ & $3 / 32(9.38 \%)$ \\
\hline Klebsiella pneumonia & $1 / 4(25 \%)$ & $0 / 4(0 \%)$ & $0 / 4(0 \%)$ \\
\hline Klebsiella oxytoca & $0 / 2(0 \%)$ & $0 / 2(0 \%)$ & $0 / 2(0 \%)$ \\
\hline Acinetobacter spp. & $0 / 5(0 \%)$ & $0 / 5(0 \%)$ & $0 / 5(0 \%)$ \\
\hline Pseudomonas aeruginosa & $1 / 3(0 \%)$ & $0 / 3(0 \%)$ & $0 / 3(0 \%)$ \\
\hline Citrobacter freundii & $0 / 1(0 \%)$ & $0 / 1(0 \%)$ & $0 / 1(0 \%)$ \\
\hline Citrobacter diversus & $0 / 3(0 \%)$ & $0 / 3(0 \%)$ & $0 / 3(0 \%)$ \\
\hline Proteus vulgaris & $1 / 1(100 \%)$ & $0 / 1(0 \%)$ & $0 / 1(0 \%)$ \\
\hline \multicolumn{1}{|c|}{ Total } & $\mathbf{2 0 / 5 1 ( 3 9 . 2 2 \% )}$ & $\mathbf{8 / 5 1 ( 1 5 . 6 9 \% )}$ & $\mathbf{3 / 5 1 ( 5 . 8 8 \% )}$ \\
\hline
\end{tabular}

Out of 51 gram negative isolates, the distribution of ESBL, Ampc-beta lactamase and co-existence of ESBL and AMPC production was found to be $39.22 \%, 15.69 \%$ and $5.88 \%$ respectively. 
AMP: resistant, COT: Resistant, NX: sensitive, NIT: sensitive, NA: sensitive, GEN: resistant, CTX: resistant, ESBL: positive, ABL: positive. MDR: strain

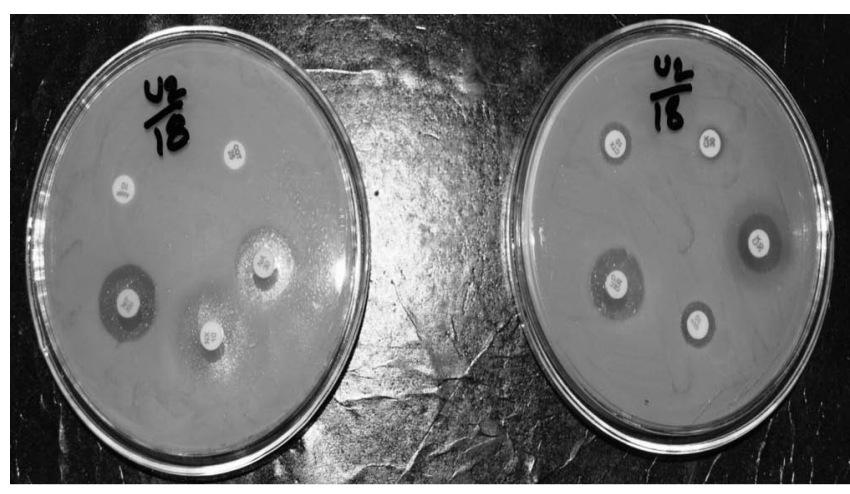

Figure 1: showing ESBL (positive), ABL (positive) and MDR

\section{DISCUSSION}

The $\beta$-lactam antibiotics are the most widely used agents and resistance to them is reported from all over the world. The most important mechanism of resistance to these $\beta$-lactam antibiotics is the production of an enzyme $\beta$-lactamase, which destroys the $\beta$-lactam ring of the $\beta$-lactam antibiotics. ${ }^{13}$

The occurrence of ESBL producers among the gram negative bacilli in the current study was 39.22\% (20/51), while 100 \% Proteus mirabilis, $53.13 \%$ E. coli, $25 \%$ Klebsiella pneumoniae, and 33.33\% Pseudomonas aeruginosa were found to elaborate ESBLs. The result of prevalence of ESBL producing gram negative bacteria were in contrast with study done by Singhal et al. ${ }^{12}$ (64\%) and Dalela et al.(66.9 \%). The ESBL production in Pseudomonas aeruginosa is less $(33.33 \%)$ as compared to that in other gram negative bacilli, because their resistance mechanism is mediated by the production of metallo beta-lactamase, lack of drug penetration due to mutations in the porins or loss of certain outer membrane proteins and an efflux pump. ${ }^{8}$

In this study, ABL production was seen in $15.69 \%(8 / 51)$ of total gram negative isolates. All ABL producers were $E$. coli. Similar finding was seen in the study done by Laghawe et al., ${ }^{13}$ (15.97\%), Bareja et al., 2013 (15.3\%), and Yusuf, Haruna, ${ }^{3}(11.3 \%)$. The prevalence of ABL production was observed in study a done by Singhal et $a .^{12}(36 \%)$ and Sridhar Rao, Leelakrishna, (50\%). ${ }^{14}$

Recently, the co-existence of both ABL and ESBL in some gram negative bacilli has also been reported. This could be because plasmid mediated $\mathrm{ABL}$ has been disseminated among the Enterobacteriaceae, sometimes in combination with ESBL. Such strains may give false negative tests in the detection of ESBL. ${ }^{8}$

In the present study co-existence of the phenotypes of both the ABL and the ESBL producers was $5.88 \%$ (3/51) and such a co-existence was observed among the E. coli isolates only. Similar, finding were observed in study done by Sridhar Rao, Leelakrishna, ${ }^{14}$ 8\%, Sasirekha et al., ${ }^{15} 6.2 \%$ Yusuf, Haruna, ${ }^{3}$ $6.04 \%$, and Dalela et al., $3.5 \%$. Overall, in this study, the prevalence of ESBL and ABL and the coexistence of the phenotype $(\mathrm{ESBL}+\mathrm{ABL})$ in the urinary isolates was found to be $39.22 \%, 15.69 \%$ and $5.88 \%$.

\section{CONCLUSION}

Antibiotic resistance is a global problem. It is now generally considered as public health issue and has significant implication in health sector. Resistance to antimicrobial drugs is causing increasing morbidity and mortality due to infectious diseases. The problem of the bacterial resistance to antimicrobial drugs is more troublesome in developing countries like Nepal.

\section{ACKNOWLEDGEMENT}

I would like to express my deep sense of gratitude to Mrs. Jyoti Acharya, Senior Medical Lab. Technologist, Sukraraj Tropical and Infectious Disease Hospital (STIDH), Kathmandu and Mrs. Archana Katuwal, Senior Lecturer, Kathmandu College of Science and Technology (KCST). Finally, I express my profound gratitude to my parents, and my brothers for their understanding, continuous inspiration, encouragement and unconditional support.

\section{REFERENCES}

1. Altun Ş, Tufan ZK, Yağcı S, Önde U, Bulut C. Extended Spectrum Betalactamases, AmpC and Metallo Beta-lactamases in emerging multi-drug resistant Gram-negative bacteria in intensive care units, 2013; 2:707.

2. Rajesh RB, Shinu SP, Shah RK, Prem PSG, Singh VA. Simultaneous detection of Extended-spectrum- $\beta$-lactamase, AmpC$\beta$-lactamase and Metallo- $\beta$-lactamase in gram negative clinical isolates on a single plate. Journal of Dental and Medical Sciences, 2013; 6(2):74-77.

3. Yusuf I, Haruna M. Detection of AMPC and ESBL Producers among Enterobacteriaceae in a Tertiary Health Care in, Kano- Nigeria. International Journal of Science and Technology, 2013; 3(4):2224-3577.

4. Thomson KS. Extended-Spectrum--Lactamase, AmpC, and Carbapenemase Issues. J Clin Microbiol, 2010; 48(4):101925.

5. Gupta K, Hooton TM, Naber KG, Wult B, Colgan R, Miller LG, Morgan GJ, Nicolle, LE, Raz R ,Schaeffer AJ, Soper DE. : International clinical practice guidelines for the treatment of acute uncomplicated cystitis and pyleonephritis in women 
: a 2010 update by the infectious diseases society of America and the European society for microbiology and infectious diseases. Clin Infect Dis, 2011; 52:e.103-120.

6. Kattel HP, Mishra SK, Acharya J, Sigdel MR, Shah NP, Shah AS, et al. Antibiotic sensitivity profile of different uropathogens in tertiary care center in Nepal. JNAMLS, 2012; 11(1):19-33.

7. Clinical and Laboratory Standards Institute/NCCLS. Performance Standards for Antimicrobial Susceptibility Testing. Nineteenth informational supplement. M100-S19. CLSI, Wayne, PA, 2009.

8. Dalela G, Gupta S, K DKJD, Mehta P. Antibiotic Resistance Pattern in Uropathogens at a Tertiary Care Hospital at Jhalawar With Special Reference to ESBL, AMPC $\beta$-Lacatamase and MRSA Production. Journal of Clinical and Diagnostic Research, 2012; 6(4):645-51.

9. Coudron PE, Moland ES, Sanders CC. Occurrence and detection of Extended spectrum beat lactamases in members of the family Enterobacteriaceae at a veterans medical center: seek and you may find. J. Clin. Microbial, 1997; 35:2593-7.

10. Grover N, Brig AKS, Bhattacharya S. Therapeutic challenges of ESBLS and AmpC- beta lactamase producers in a tertiary care center. Medical Journal Armed Force India, 2013; 69:4-10.

11. Gopal DVRS, Kumar E, Usha K, BV BVR, Chaudhary A. Prevalence of various $\beta$-lactamase (ESBL, AMPC AND MBL) producing multidrug resistant clinical isolates of Acinetobacter spp. in a tertiary care hospital. Asian J Pharm Clin Res, 2013; 6:28-31.

12. Singhal S, Mathur T, Khan S, Upadhyay DJ, Chugh S, Gaind R, et al. Evaluation of methods for Ampc- $\beta$ lactamase in gram negative clinical isolates from tertiary care hospitals. Indian Journal of Medical Microbiology, 2005; 23(2):120-4.

13. Laghawe AR, K MJN, Thombare VR. Prevalence of AMPC Beta-lactamase in Gram- negative bacilli. Journal of Pharmaceutical and Biomedical Sciences, 2012; 20(20):1-4.

14. Sridhar RPN, Leelakrishna G, Basavarajappa KG. Detection of extended spectrum beta-lactamase from clinical isolates in Davangere. Indian J Pathol Microbiol, 2006; 51(4):497-9.

15. Sasirekha B. Prevalence of ESBL, AMPC B- LACTAMASE and MRSA among uropathogens and its antibiogram. EXCLI J, 2013; 12:81-8. 